Ann. Biol. anim. Bioch. Biophys., I973, 13 (3), 48I-503

\title{
UNE MÉTHODE D'ANALYSE GRAPHIQUE DES COURBES DE CROISSANCE
}

\author{
E. SCHIFFLERS ( $\mathbf{1}$ \\ avec la collaboration technique de Renée VINCK \\ Laboratoire de Génétique F. A. JANSSENS \\ Faculté des Sciences agronomiques, \\ Université catholique de Louvain (Belgique)
}

\section{RÉSUMÉ}

Cette étude a pour objet l'analyse des courbes de croissance en poids des souris de laboratoire. Les souches consanguines $\mathrm{C}_{3} \mathrm{H}$ et $\mathrm{C}_{57} \mathrm{BL}$ ainsi que leurs hybrides réciproques ont fourni le matériel expérimental. Les courbes d'animaux d'un même groupe sont dessinées par ordinateur dans un mếme graphique. Les dessins obtenus permettent une première analyse qualitative du phénomène. Il est possible de distinguer l'enfance, l'adolescence et l'âge adulte sur les graphiques. Les caractéristiques des deux premières phases sont décrites pour les différents groupes étuđiés. Les résultats sont comparés aux techniques usuelles de description de la croissance.

\section{I. - INTRODUCTION}

Les biologistes qui étudient la croissance des animaux accumulent généralement de grandes quantités de données expérimentales. L'abondance des mesures rend un résumé nécessaire. Nous proposons dans le présent travail une méthode graphique simple qui permet de visualiser un ensemble de résultats expérimentaux de croissance. La technique que nous présentons ici consiste à faire dessiner par ordinateur différentes courbes de croissance dans un même graphique.

Les expérimentateurs utilisent classiquement d'autres techniques. La plus élémentaire consiste à représenter les données par la courbe des moyennes de la grandeur mesurée. On y ajoute souvent l'écart-type et d'autres paramètres statistiques simples. Entre autres Young et al. (I965), BUTLER et METrakos (I950), ont utilisé l'analyse de variance pour étudier les influences génétiques et maternelles dans la croissance des souris. On peut aussi ajuster une courbe théorique aux données expérimentales. On trouvera un exposé des différentes courbes les plus utilisées dans le travail de Von BERTALANFFY (I960). Plus récemment, et pour citer des exemples

(1) Adresse actuelle : Centre de Calcul, Maxwell, Sciences 3, B. I348 Louvain la Neuve, Belgique. 
relatifs aux souris, LAIRD (I967) utilisait la courbe de Gompertz pour décrire la croissance en poids de diverses souches de souris. GALI, et KYLE (Ig68), quant à eux; se servent de la courbe de von Bertalanffy pour une description identique. Enfin KIDWELI et al. (I969 et I970) analysent 1'hérédité de la croissance des souris au moyen d'une description polynomiale d'une part et d'une description par des courbes de Gompertz de 1'autre. On comprend dès lors la perplexité de l'expérimentateur devant le choix d'une méthode d'analyse de ses courbes de croissance. Aussi le présent travail se propose-t-il de fournir une étude méthodologique qui permettrait aux chercheurs de choisir plus aisément une méthdode d'analyse des courbes de croissance. Pour cela, nous confronterons nos résultats à ceux qui ont été obtenus antérieurement par d'autres méthodes. Nous constaterons que les faits mis en évidence par les graphiques superposés confirment en général les observations antérieures concernant la croissance. En conclusion, nous pourrons voir que l'analyse de variance, les courbes de moyennes et écarts-types apportent un complément quantitatif à la méthode des courbes superposées, mais que cette dernière est nécessaire à l'interprétation des méthodes citées. Nous verrons aussi que les modèles usuels ne rendent pas compte de tous les faits observés sur les courbes de croissance superposées.

\section{II. - MATÉRIEL E'T MÉTHODES}

\section{I. - Matériel}

Les souches initiales étaient constituées de souris consanguines (reproduites par inceste frère-sœur) $\mathrm{C}_{3} \mathrm{H}$ et $\mathrm{C} 57 \mathrm{BL}$ provenant de l'animalerie de l'Université de Louvain, et élevées pendant trois générations au moins dans le laboratoire d'expérience avant de fournir les individs $\mathbf{s}$ mesurés. Les premiers sujets étudiés étaient des $\mathrm{C}_{3} \mathrm{H}$ et des $\mathrm{C} 57 \mathrm{BL}$; ils forment la génération o. La génération I a été constituée des enfants de la génération o; elle contient quatre types de souris : $\mathrm{CC}$ (issues d'un croisement frère-sœur $\mathrm{C}_{3} \mathrm{H}$ ), $\mathrm{BB}$ (croisement frère-sœur $\mathrm{C} 57 \mathrm{BL}$ ), $\mathrm{CB}$ (croisement mère $\mathrm{C}_{3} \mathrm{H}$ et père $\mathrm{C}_{57} \mathrm{BL}$ ), $\mathrm{BC}$ (croisement mère $\mathrm{C}_{57} \mathrm{BL}$ et père $\mathrm{C}_{3} \mathrm{H}$ ). A l'avenir nous appellerons groupe un ensemble de souris nées dans une même génération d'un même type de croisement et de même sexe. Le tableau I donne les effectifs de chaque groupe expérimental,

TABLEAU I

Groupes et effectifs expérimentaux

\begin{tabular}{l|c|c|c|c}
\hline \hline Nom & Génération & \multicolumn{1}{|c|}{ Origine } & \multicolumn{2}{|c}{ Effectifs } \\
& & & Mâles & Femelles \\
\hline C 57 BL & 0 & Stock inbred & 25 & 22 \\
C 3 H & 0 & Stock inbred & 20 & 27 \\
CC & 1 & Inbred C 3 H & 17 & 21 \\
CB & 1 & Hybrides C 3 H $\times$ C 57 BL & 17 & 23 \\
BC & 1 & Hybrides C 57 BL $\times$ C 3 H & 37 & t0 \\
BB & 1 & Inbred C 57 BL & 16 & 20 \\
\hline \hline
\end{tabular}

$$
\text { 2. 2. - Méthode }
$$

Les conditions d'élevage ont été maintenues constantes dans la mesure du possible. Les facteurs suivants étaient contrôlés : température $\left(25^{\circ} \mathrm{C} \pm \mathrm{I}^{\circ} \mathrm{C}\right.$ ), humidité (minimum de $60 \mathrm{p}$. Ioo d'humidité relative), photopériode (lumière de 7 à $19 \mathrm{~h}$ ). L'eau et la nourriture étaient distribués 
à volonté. Nous n'avons aucune indication sur les variations de la composition des aliments commerciaux utilisés durant l'expérience.

Chaque souris était pesée quotidiennement depuis sa naissance (jour o) jusqu'au jour I2o. En raison du cannibalisme fréquent chez les mères $\mathrm{C}_{3} \mathrm{H}$ et $\mathrm{C} 57 \mathrm{BL}$, les animaux d'expérience étaient placés en nourrice chez des femelles NMRI, le matin de leur naissance. Dans la mesure du possible les nichées contenaient six individus. Le sevrage avait lieu au matin du jour 21 . Les accouplements étaient effectués après le $120^{\mathrm{e}}$ jour.

\section{3. - Calculs et dessins}

L'analyse des résultats repose sur une technique qui consiste à superposer dans un même graphique les courbes relatives aux individus d'un même groupe ou d'une même famille. Le traitement numérique et graphique a été réalisé par un ordinateur (IBM I620) relié à une table traçante (IBM I627 mod II). Nous disposions d'une bibliothèque de sous-routines ForTRAN II conçues pour les dessins. Les programmes rédigés à cette occasion sont disponibles; ils n'ont pas été reproduits ici étant donné qu'ils sont limités par les caractéristiques des machines utilisées.

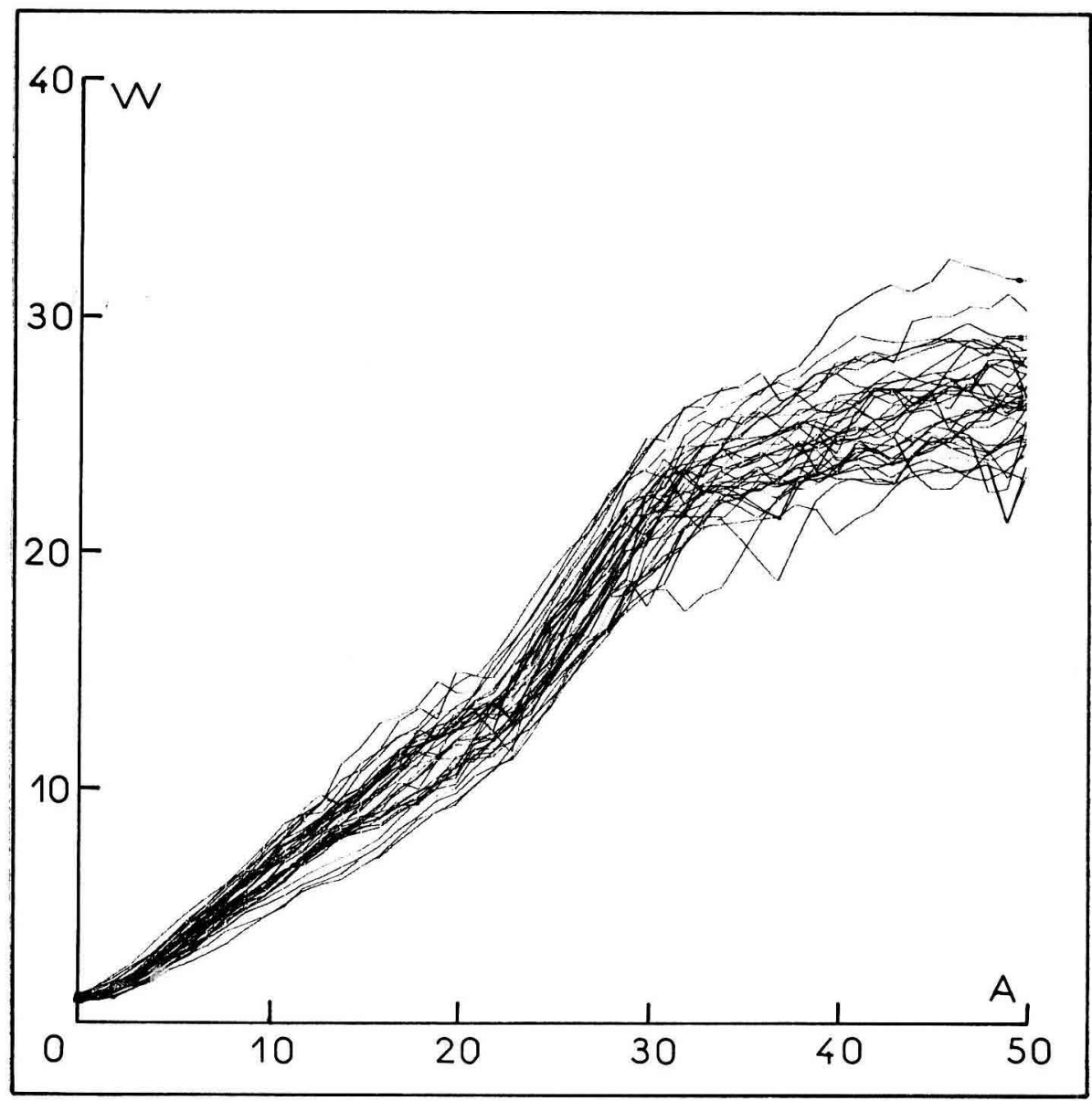

FIG. I. I. - Poids superposés des mâles $B C$ (37 courbes)

FIG. DE LA SÉRIE I. - Courbes superposées de poids

Chaque figure rassemble dans un même graphique les courbes de poids en fonction de l'âge pour les individus d'un même groupe expérimental. Le poids W est exprimé en grammes, et l'âge A en jours. L'enfance et l'adolescence sont entièrement représentées. 
E. SCHIFFLERS

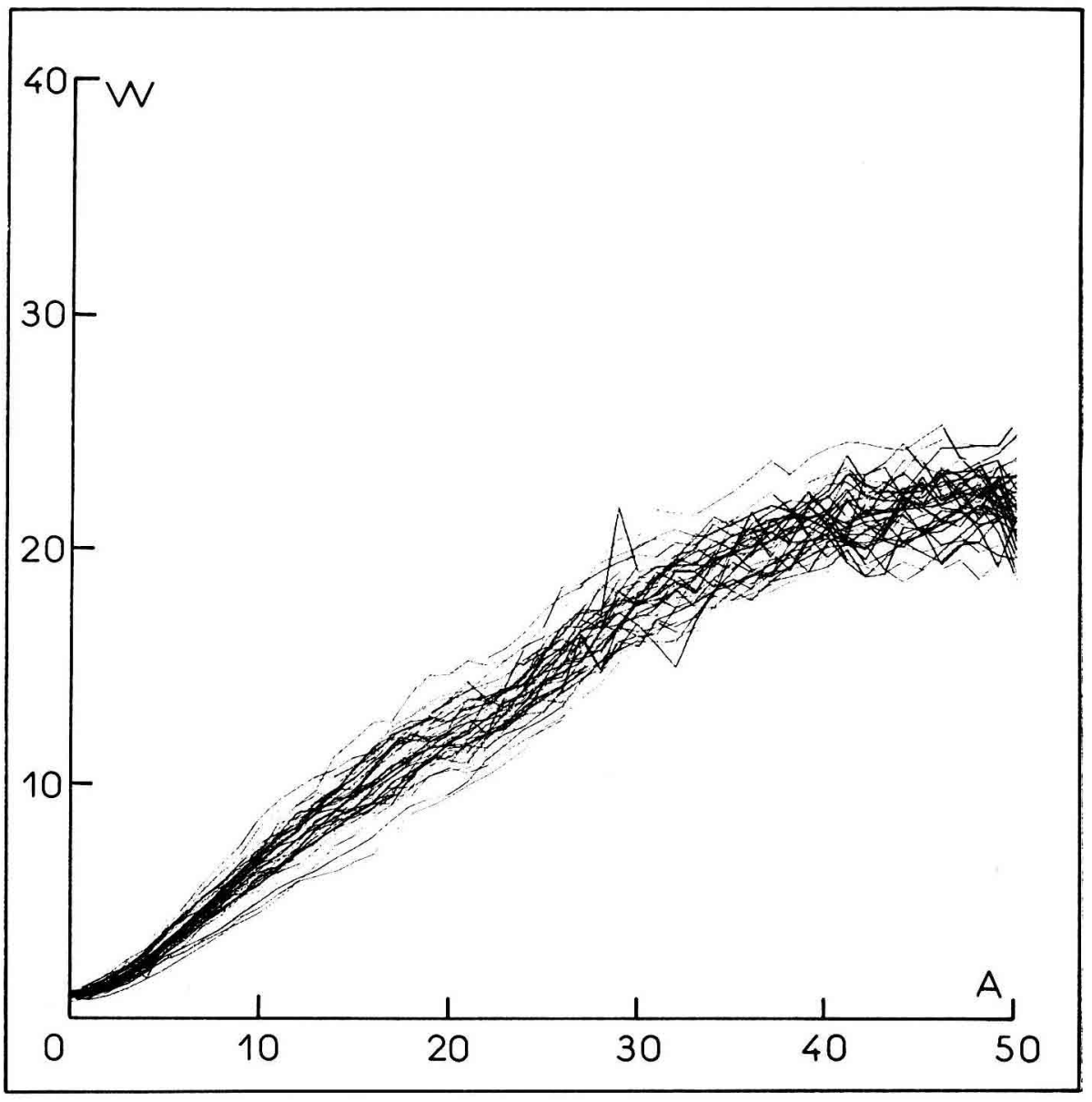

FIG. I. 2. - Poids superposés des femelles $B C$ (40 courbes) 


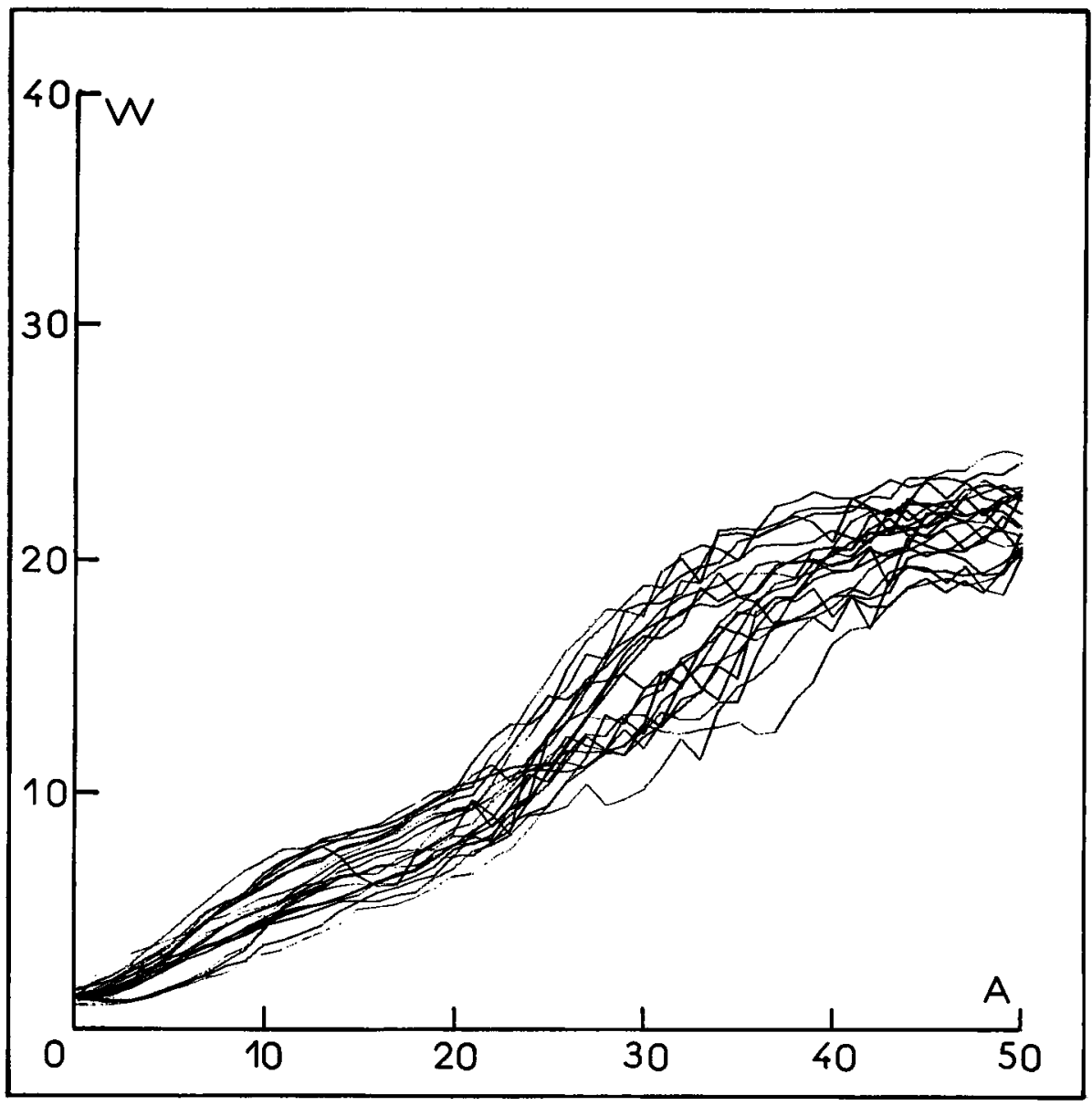

FIG. 1. 3. - Poids superposés des males $C 3 H$ (20 courbes) 


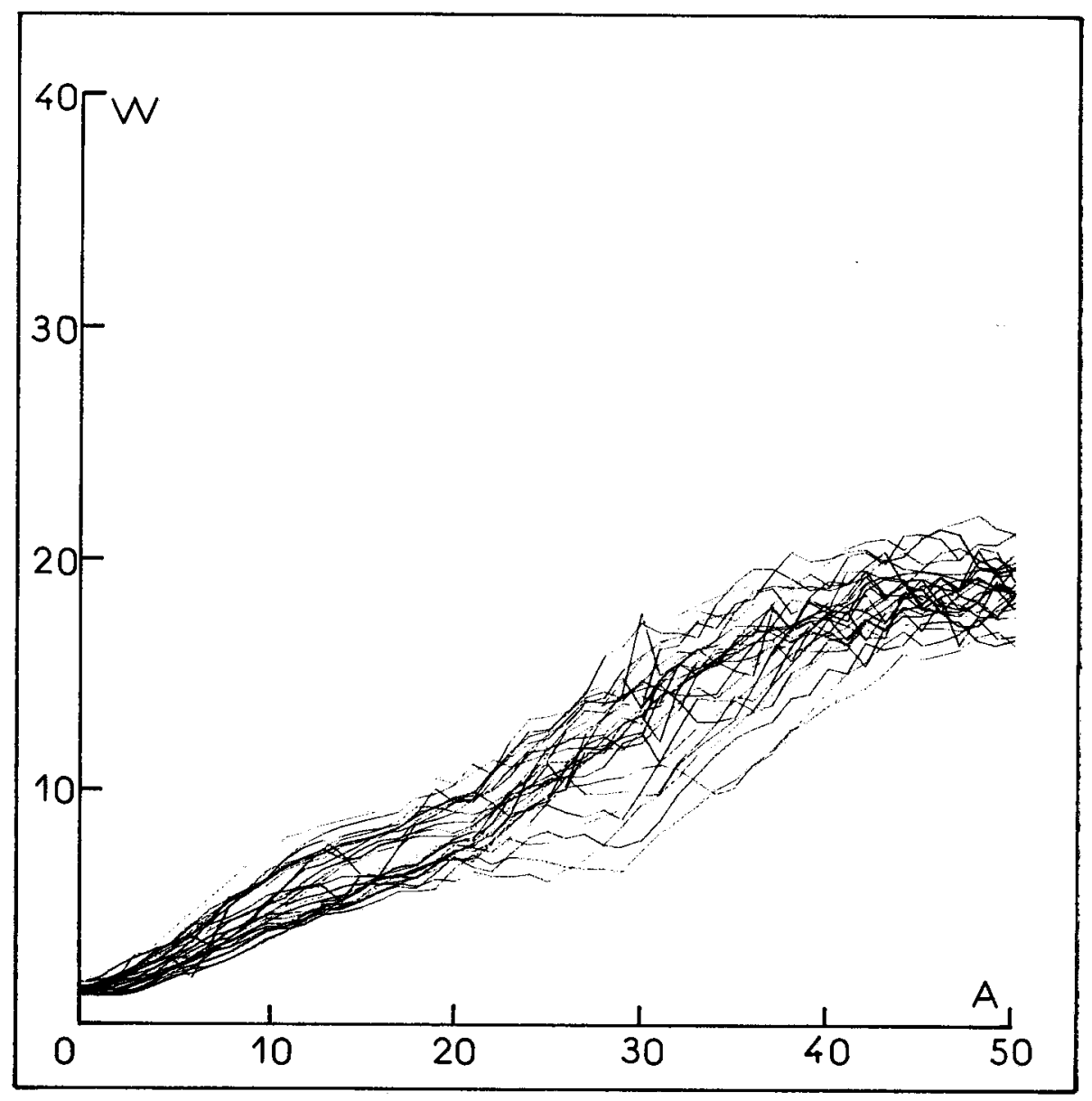

FIg. I. 4. - Poids superposés des femelles $C 3 H$ (27 courbes) 


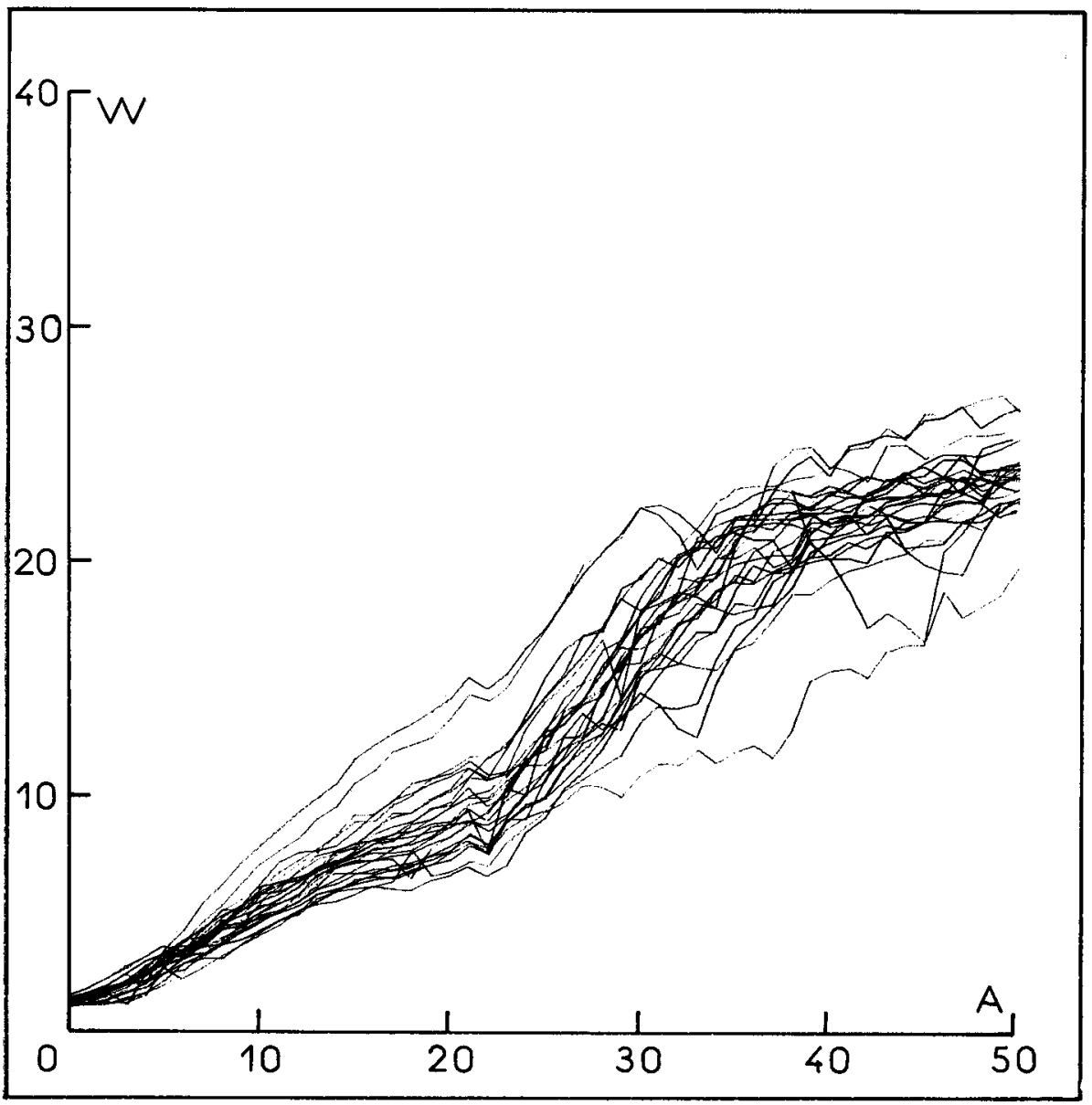

FIG. I. 5. - Poids superposés des mâles C 57 BL (25 courbes) 
E. SCHIFFIERS

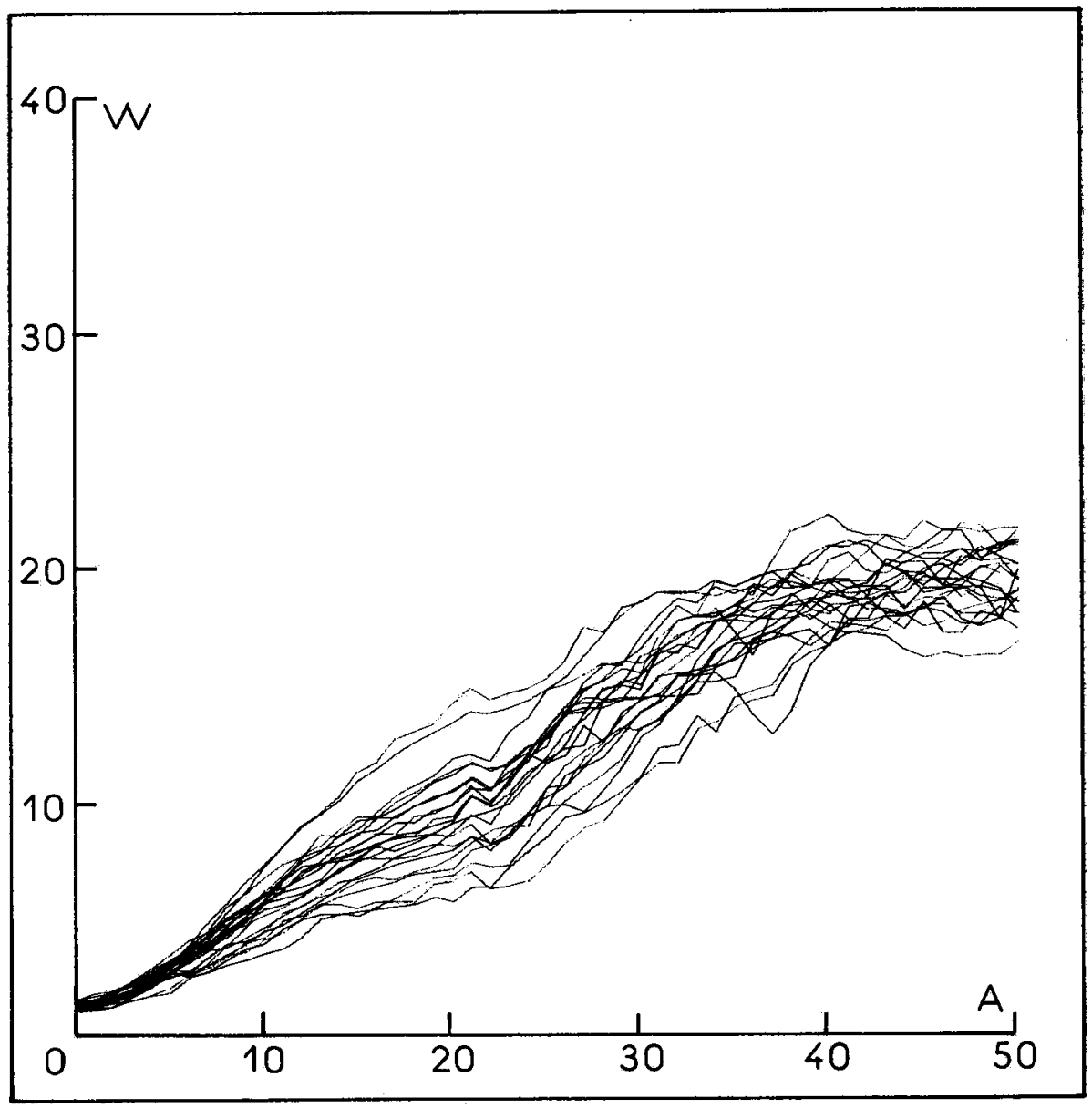

FIG. I. 6. - Poids superposés des femelles $C 57$ BL (22 courbes) 


\section{III. - RÉSULTATS}

\section{I.}

Dans l'exposé des résultats nous emploierons différents termes que nous précisons ici. Outre la forme générale des graphiques, nous attacherons notre attention aux caractéristiques suivantes.

a) Dans chaque figure de la série I par exemple, nous voyons une zone dans laquelle la majorité des courbes de croissance s'enchevêtrent plus ou moins. Nous donnerons à cette zone le nom de " bande noire " du graphique.

b) La dispersion des courbes au jour $\mathrm{j}$ est l'épaisseur de la bande noire à ce jour. En fait nous ne désirons pas mesurer cette épaisseur; nous nous intéresserons surtout aux variations de la dispersion au cours du temps.

c) Dans certains cas, il apparaitra que la bande noire du graphique se subdivise en faisceaux ainsi que 1'illustre la figure I. 4 aux alentours du jour 17 .

d) Les oscillations d'une courbe individuelle sont les variations quotidiennes de sa valeur.

\section{2.}

Six exemples de courbes superposées de poids sont représentés ici (figures de la série r). Chacun d'eux est obtenu en dessinant sur un même graphique les courbes de poids des animaux d'un même groupe expérimental. Durant l'enfance (o à $20 .$. 25 jours), la dispersion augmente régulièrement. Durant l'adolescence (20... 25 à $35 \ldots 40$ jours) la bande noire montre une croissance générale plus forte que dans l'enfance. La dispersion augmente toujours durant cette période. L'âge adulte n'est pas représenté ici (40 à I20 jours) pour les raisons invoquées plus haut. Disons seulement ici que la dispersion augmente jusqu'au jour I20, après avoir diminué entre le jour 35 et 40 . Toutes les souris continuent à croître en poids durant l'âge adulte. I Les différences les plus remarquables entre les groupes peuvent se résumer comme suit. Les groupes mâles (fig. I. I, I. 3, I. 5) accusent une bande noire très croissante durant l'adolescence. Les groupes femelles (fig. I. 2, I. 4, I. 6) montrent les mêmes caractéristiques bien que la croissance à l'adolescence soit moins forte que chez les mâles. Les hybrides (fig. I. I et I. 2) se distinguent des consanguins (fig. I. 3, I. 4, I. 5, r. 6) par une dispersion plus faible chez les premiers. La dispersion des consanguins s'explique soit par la présence de plusieurs faisceaux (fig. I. 3 et I. 4), soit par de grandes différences entre les courbes individuelles. Enfin, dans l'ensemble, les oscillations des courbes individuelles sont d'amplitude plus faible que les différences entre courbes.

L'examen de toutes les courbes superposées de poids confirmerait la description faite ci-dessus.

\section{3 .}

Les deux phases que nous avons décrites ci-dessus (enfance et adolescence) apparaissent plus nettement sur les graphiques des accroissements de poids que l'on peut voir aux figures 2. I. et 2.2. Nous y retrouvons les caractéristiques décrites pour les courbes de poids. On constate en effet que la figure 2. I présente deux bosses 


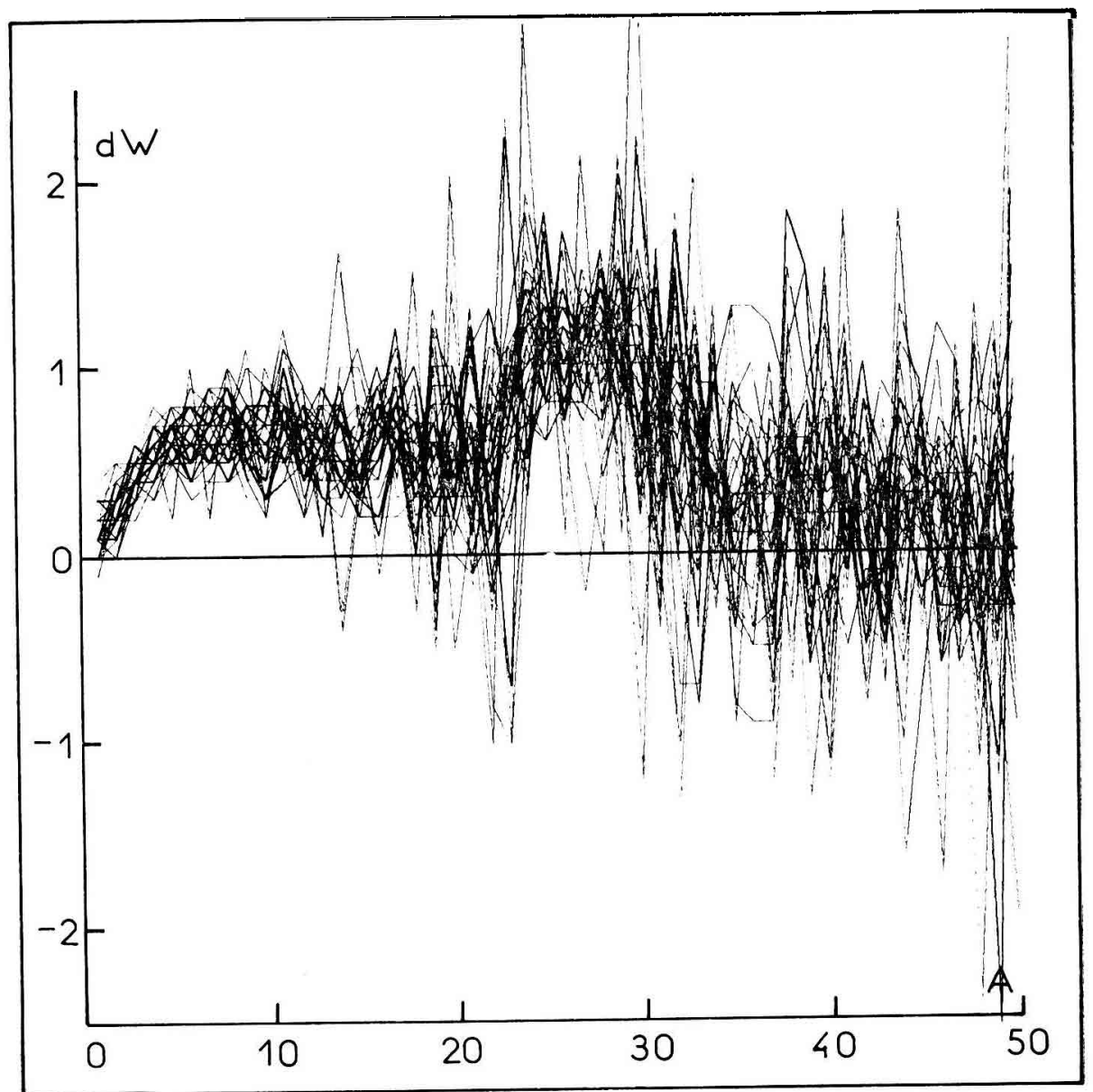

FıG. 2. I. - Accroissements de poids superposês des mâles $B C$ (37 courbes)

FIG. DE LA SÉRIE 2. - Courbes superposées des accroissements de poids

Chaque figure rassemble dans un mêrne graphique les courbes d'accroissements de poids ( $d W$ ) en fonction de l'âge $\mathrm{A}$, pour les individus appartenant à un même groupe expérimental. Les accroissements sont exprimés en grammes, l'âge en jours. 


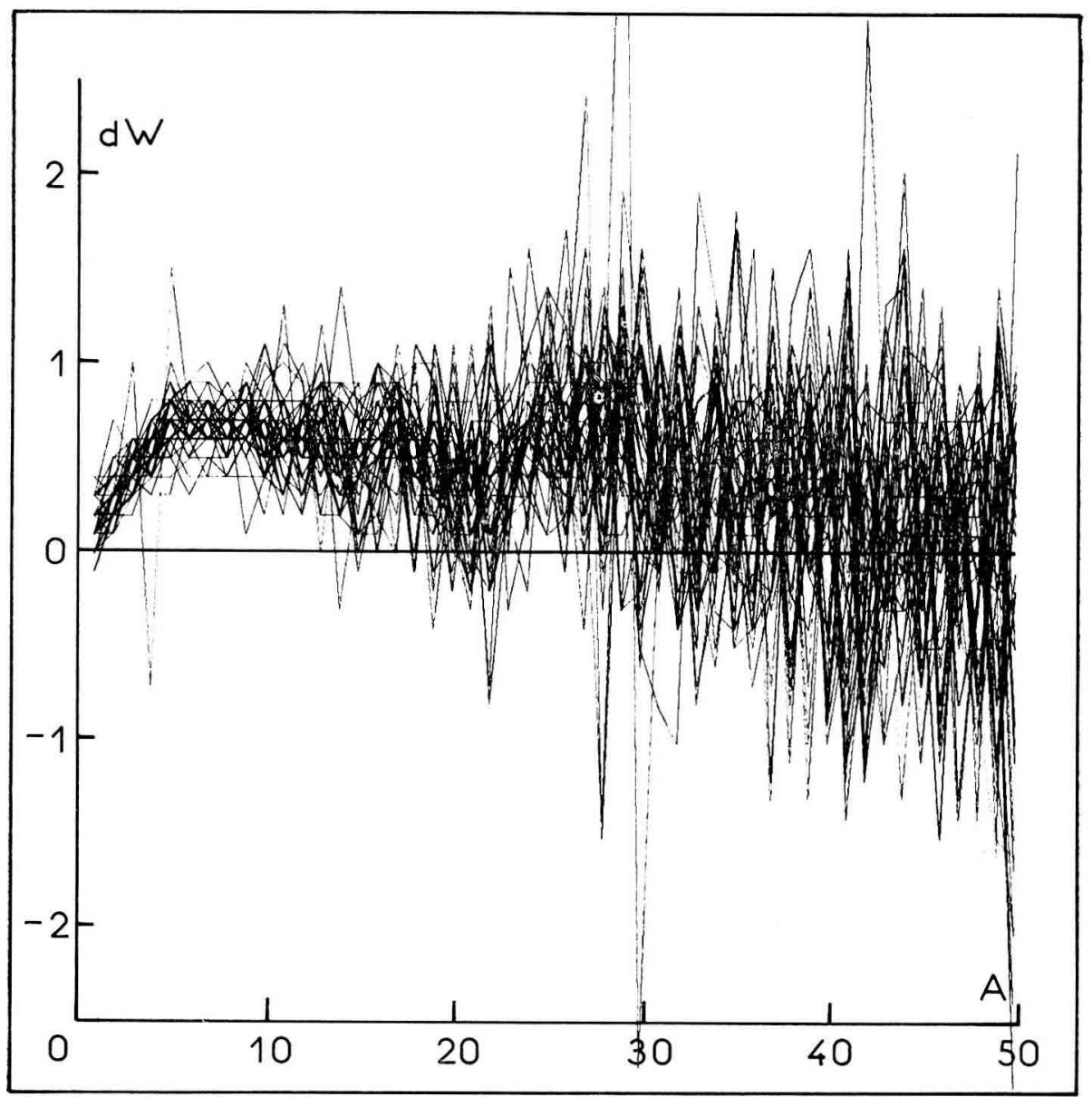

FIG. 2. 2. - Accroissements de poids superposés des femelles $B C$ (4o courbes) 


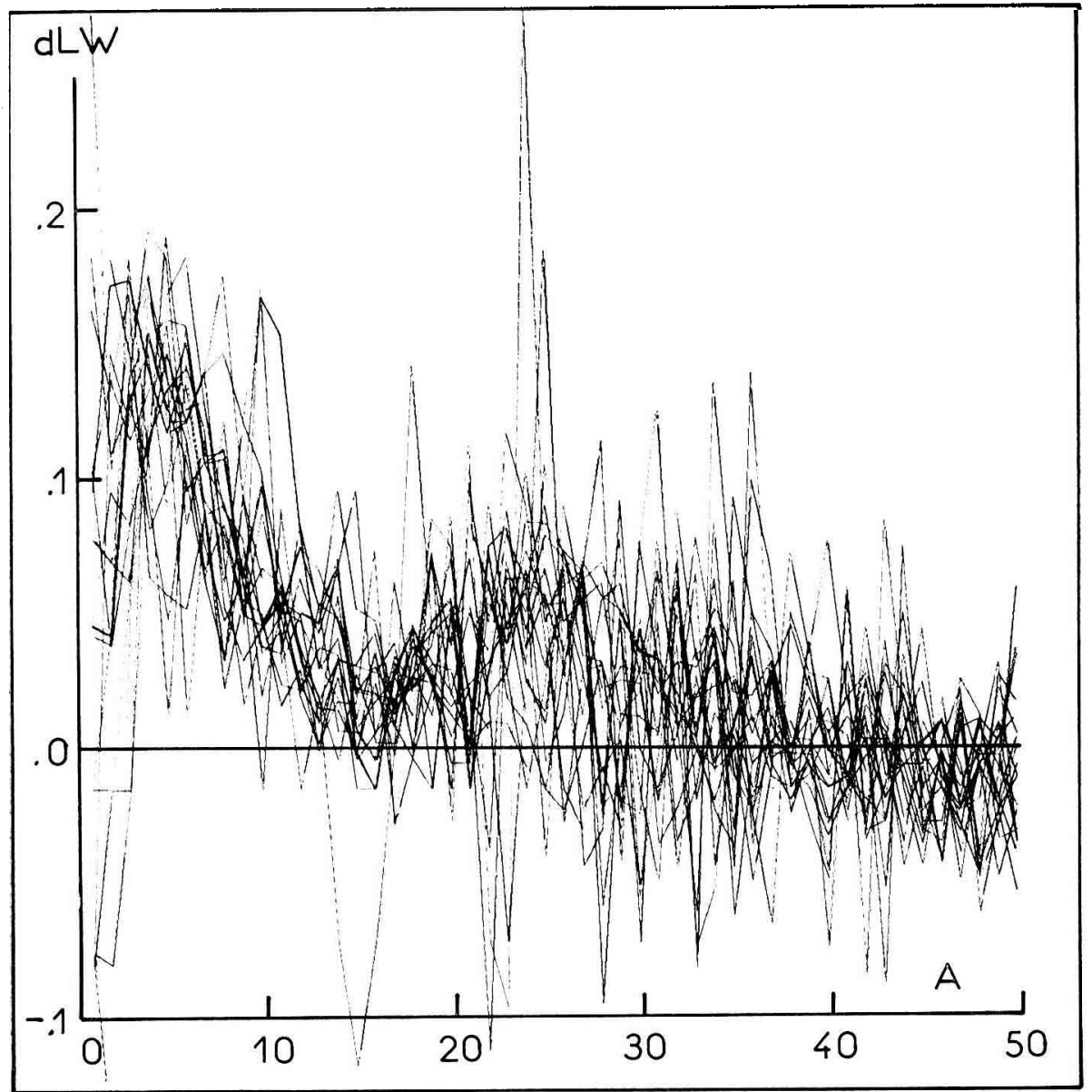

FIG. 3. I. - Accroissements logarithmiques des males $\mathrm{C} 3 \mathrm{H}$ (2o courbes)

FIG. DE LA SÉRIE 3. - Courbes superposées des accroissements du logarithme du poids

Chaque figure rassemble dans un même graphique les courbes des accroissements du logarithme du poids (dLW) en fonction de l'âge (A), pour les individus appartenant à un même groupe expérimental. L'accroissement logarithmique est l'accroissement du logarithme népérien du poids (en décigrammes) entre le jour $j$-I et le jour $j$. L'âge est exprimé en jours. 


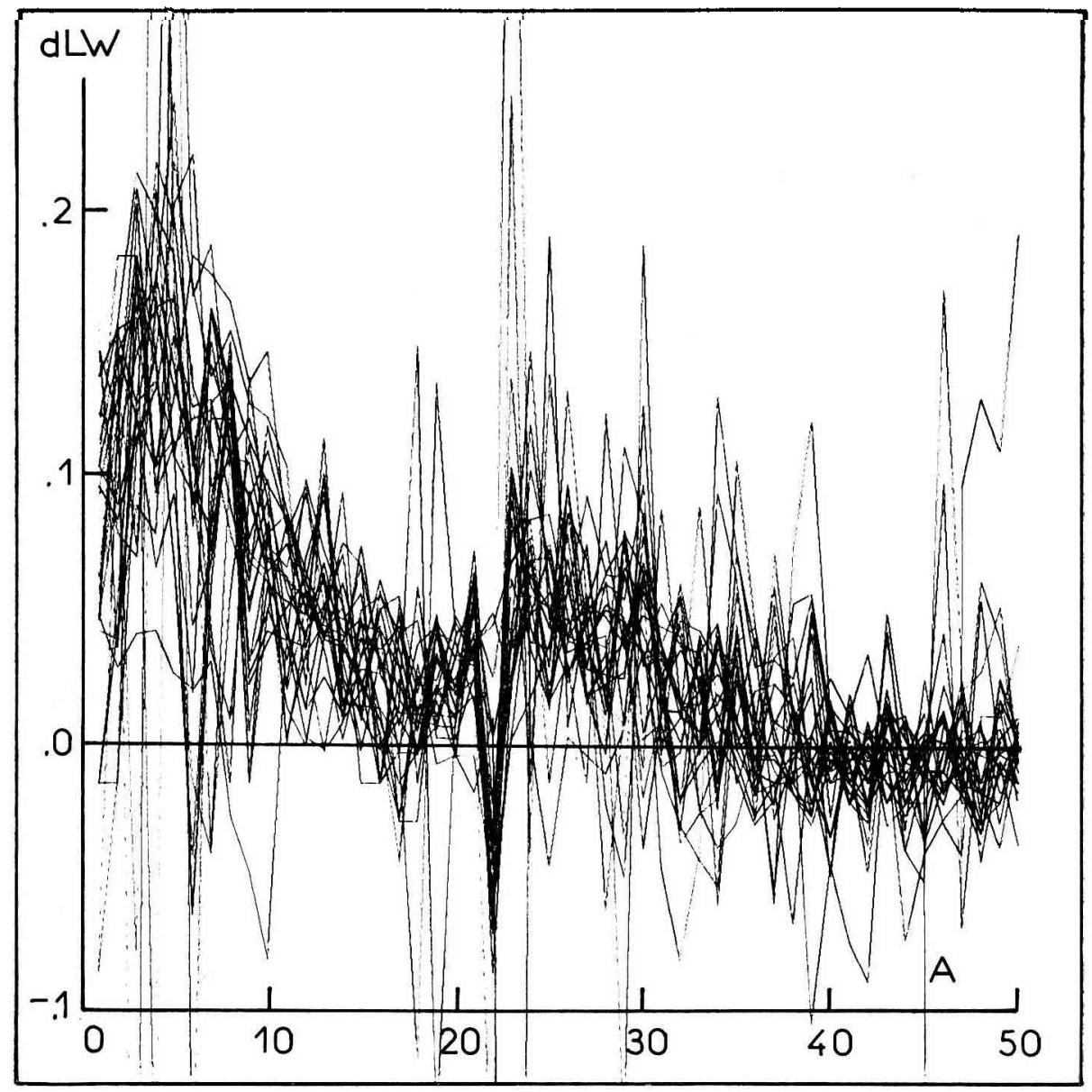

FIG. 3. 2. - Accroissements logarithmiques des mâles $C 57 B L$ (25 courbes) 
correspondant à l'enfance et 1'adolescence. La bande noire de la figure 2.2 fait aussi deux bosses, mais la seconde est nettement moins marquée que dans le graphique précédent. La dispersion des courbes est surtout due aux oscillations individuelles qui sont plus amples que les différences entre courbes.

\section{4 .}

Les figures 3. I et 3. 2 représentent des courbes superposées des accroissements du logarithme du poids. Dans la suite, nous parlerons d'accroissement logarithmique pour la grandeur obtenue en calculant la valeur de :

$$
\ln \left(w_{t} / w_{t-1}\right)
$$

où $w_{t}$ est le poids au jour $t$. Ces graphiques offrent une autre représentation de la croissance, que l'on pourrait, dans certains cas, substituer aux accroissements de poids. On constate ici que le pic d'adolescence est moins prononcé que celui de l'enfance et que la dispersion est plus stable que dans le cas des accroissements de poids. Un accident remarquable apparaît au jour 22 sur la figure 3.2 : on voit en effet que presque tous les individus maigrissent (accroissement logarithmique négatif) entre le jour 2 I et 22, c'est-à-dire au lendemain du sevrage. L'examen de tous les groupes étudiés montre que cet accident se répète dans les groupes $\mathrm{BB}$ et $\mathrm{C}_{57} \mathrm{BL}$. Dans les autres groupes, le sevrage est suivi d'une diminution de l'accroissement de poids (ou de l'accroissement logarithmique), mais rarement d'une diminution du poids.

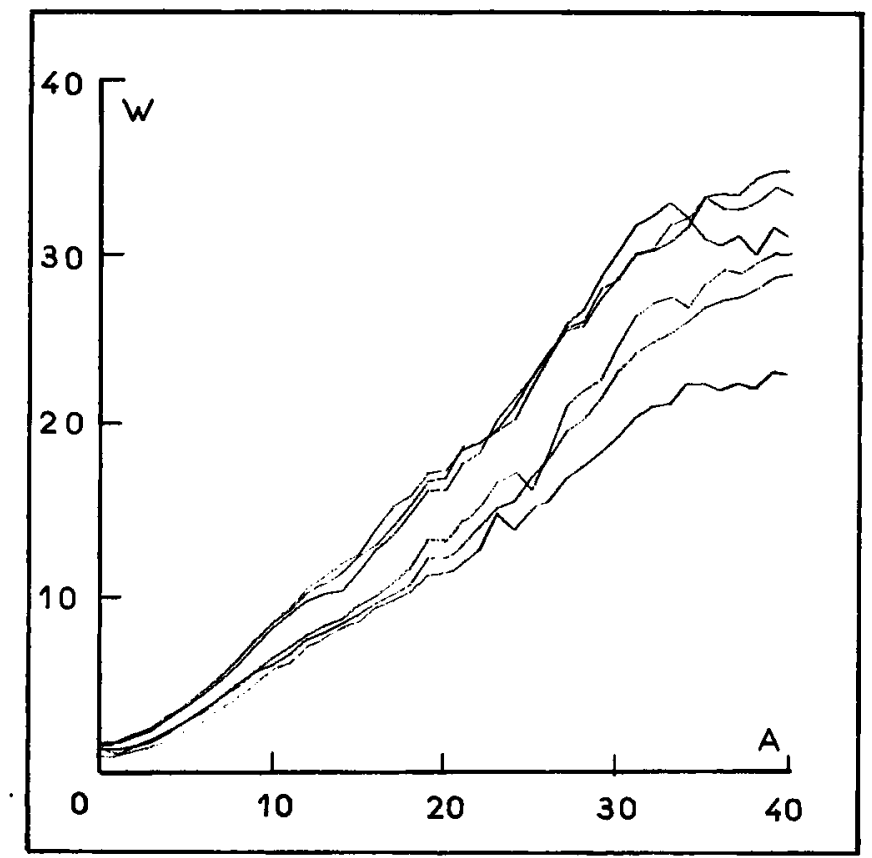

Fic. 4. I. - Courbes superposées de poids de trois consanguins $C C$ et de trois hybrides $C B$. Jusqu'au jour 21 ces six animaux ont eu une même nourrice Les trois courbes supérieures appartiennent aux hybrides

FIG. DE LA SÉRIE 4. - Courbes superposées de poids

Le poids $W$ est exprimé en grammes et l'âge A en jours. On observera sur ces courbes les regroupements durant les $2 \mathrm{r}$ premiers jours. Les individus représentés sur un même graphique ont entre eux des relations familiales explicitées dans les légendes. 


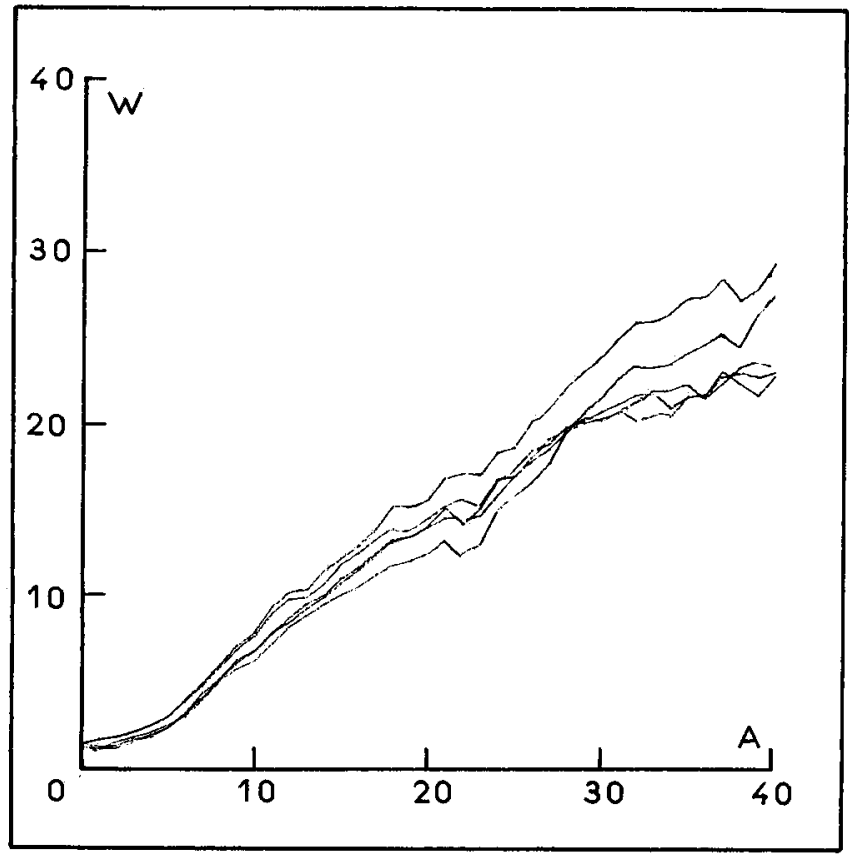

Fìg. 4. 2. - Courbes superposées de poids de cinq consanguins CC d'une même mère, placés chez deux nourrices différentes jusqu'au jour 21

Les courbes se regroupent selon l'appartenance à l'une ou l'autre nichée

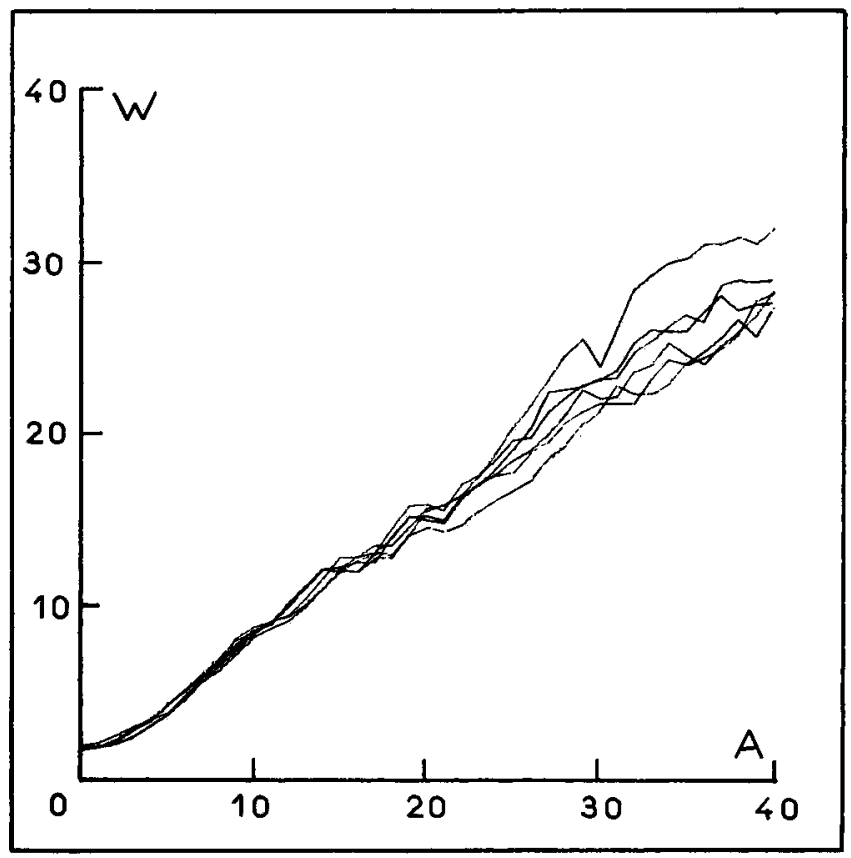

FIG. 4. 3. - Courbes superposêes de poids de six consanguins

de deux mères différentes placès chez une même nourrice jusqu'au jours 21

Les courbes se superposent indépendamment de l'appartenance à l'une ou l'autre mère Annales de Biologie animale. - r973. 


\section{$3 \cdot 5 \cdot$}

Trois exemples de courbes groupées par famille sont rassemblés à la série 4 des figures. Dans le premier exemple (fig. 4. I), on a représenté les courbes de trois souris consanguines de même mère et de troìs souris hybrides de même mère; toutes les six ont été élevées par une même nourrice. Dans le second exemple (fig. 4. 2, on trouve les courbes superposées de cinq consanguins de même mère élevés chez des nourrices différentes. Enfin, le dernier exemple (fig. 4.3) montre les courbes superposées de six individus consanguins de deux mères différentes, élevés par une même nourrice. Dans le premier cas, les trois courbes supérieures appartiennent aux hybrides, les trois autres aux consanguins. Jusqu'au dixième jour pour les consanguins et jusqu'au douzième pour les hybrides, les courbes se superposent très étroitement. On reconnaît les courbes de croissance des mâles à la grande vitesse de croissance

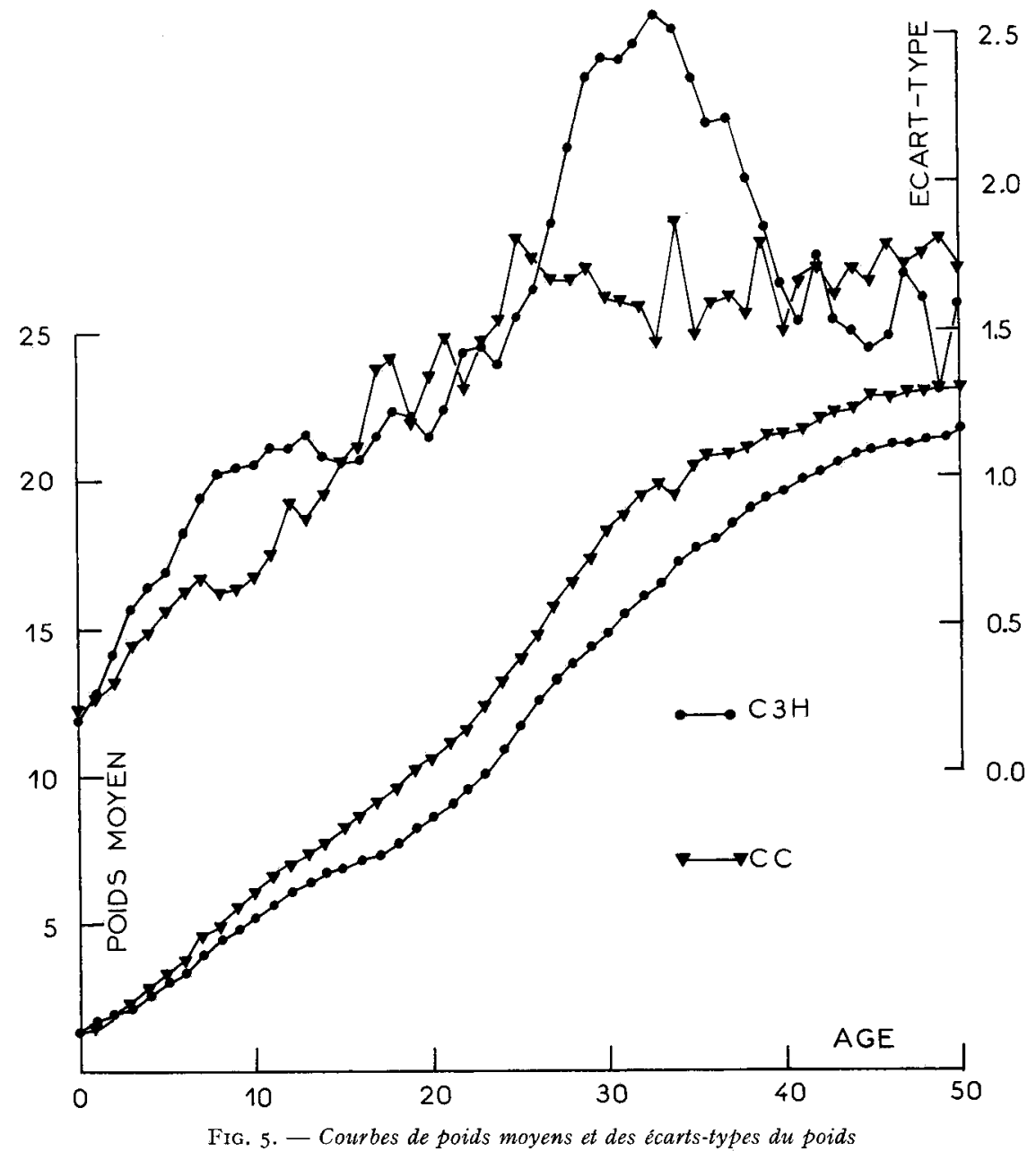

Les courbes inférieures représentent les poids moyens des mâles $\mathrm{C}_{3} \mathrm{H}$ et $\mathrm{CC}$. Le poids est exprimé en grammes. Les écarts-types correspondants sont représentés par les deux courbes supérieures. L'unité est aussi le gramme, mais l'échelle est dix fois plus précise que celle utilisée pour les poids moyens. 
après le jour 20. La figure 4. 2 montre également deux faisceaux de courbes étroitement superposées jusqu'au douzième jour environ. Chaque faisceau correspond à une nourrice. Ia figure 4.3 ne montre qu'un seul amas de courbes pendant les douze premiers jours. Naturellement les trois exemples rapportés ici sont représentatifs de la situation générale.

\section{6.}

Les courbes des poids moyens des souris mâles $\mathrm{C}_{3} \mathrm{H}$ et mâles $\mathrm{C}_{57} \mathrm{BL}$ sont représentées à la figure 5 ainsi que les écarts-types des poids des mêmes groupes. On constate que les $\mathrm{C}_{3} \mathrm{H}$ ont un poids inférieur en moyenne aux $\mathrm{CC}$. La courbe des écarts-types croît assez régulièrement avec l'âge pour les mâles $\mathrm{CC}$ entre le jour o et le jour $2 \mathrm{I}$; après quoi elle oscille autour d'une valeur sensiblement constante. Par rapport à cette courbe, les écarts-types des mâles $\mathrm{C}_{3} \mathrm{H}$ forment deux bosses. La première, peu importante, se situe de part et d'autre du dixième jour ; la seconde, beaucoup plus marquée, entre le $25^{\mathrm{e}}$ et le $40^{\mathrm{e}}$ jour. Les autres groupes de la génération $o$ présentent des courbes d'écarts-types partout très voisines de la courbe que nous avons reproduite ici. En génération I, pour les consanguins comme pour les hybrides, le modèle des courbes d'écarts-types est identique à celui des mâles CC.

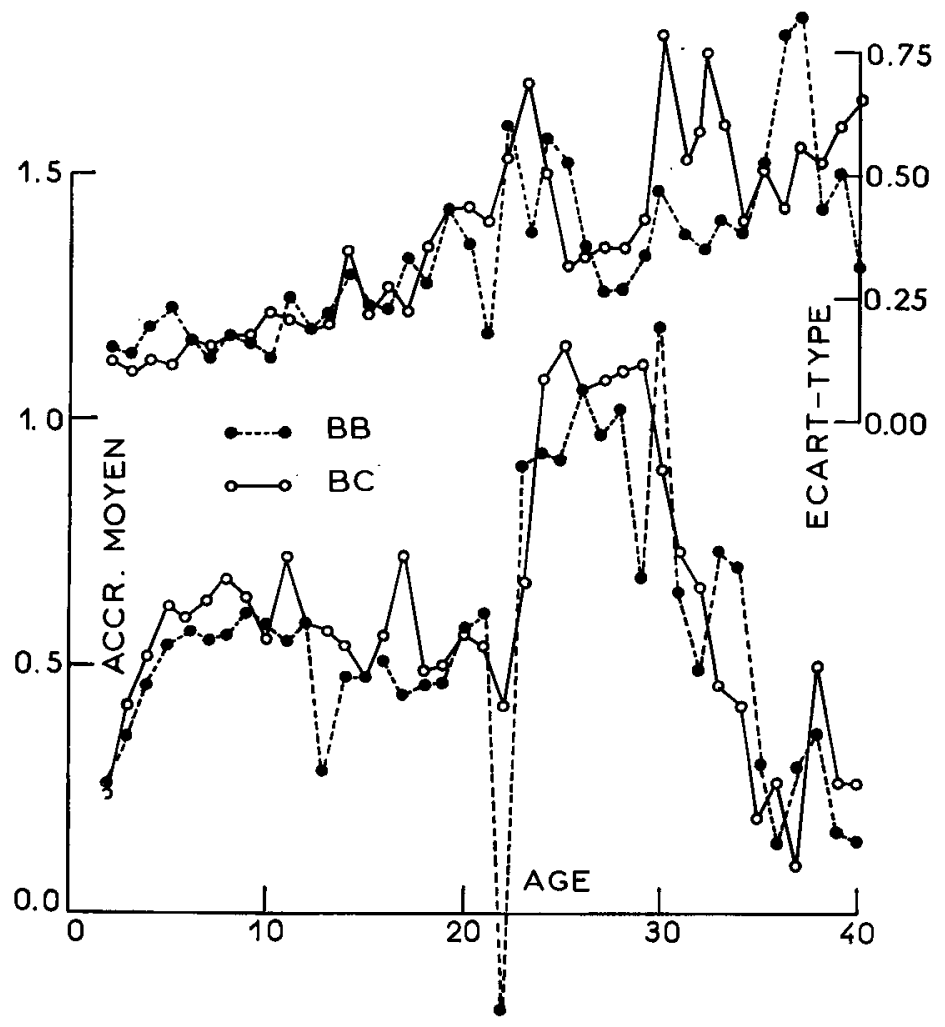

Fig. 6. - Courbes des accroissements moyens et des écarts-types

Les courbes inférieures représentent la moyenne des accroissements de poids des mâles BB et BC. L'accroissement de poids est exprimé en grammes. Les courbes supérieures utilisent la même échelle; elles représentent les écarts types correspondants. 


\section{7 .}

La figure 6 représente des courbes d'accroissements moyens des poids et les écarts types correspondants, pour les mâles $\mathrm{BB}$ et $\mathrm{BC}$. La croissance des hybrides est en moyenne plus importante que celle des consanguins. On notera la diminution du poids au jour 22 chez les BB. L'adolescence est comparable pour les deux groupes, l'intensité du phénomène étant plus grande chez les hybrides. Les écarts-types correspondant à ces courbes augmentent avec l'âge; il y a cependant, en général, une diminution de l'écart-type entre 1 e $25^{\mathrm{e}}$ et le $3^{\mathrm{e}}$ jour.

\section{8.}

Quelques courbes d'accroissements logarithmiques moyens sont représentées à la figure 7. Un premier pic se termine aux alentours du jour I4 pour les $B C$ et du jour I 2 pour les $\mathrm{CC}$. Le second pic commence aux alentours du jour 20 , après une période

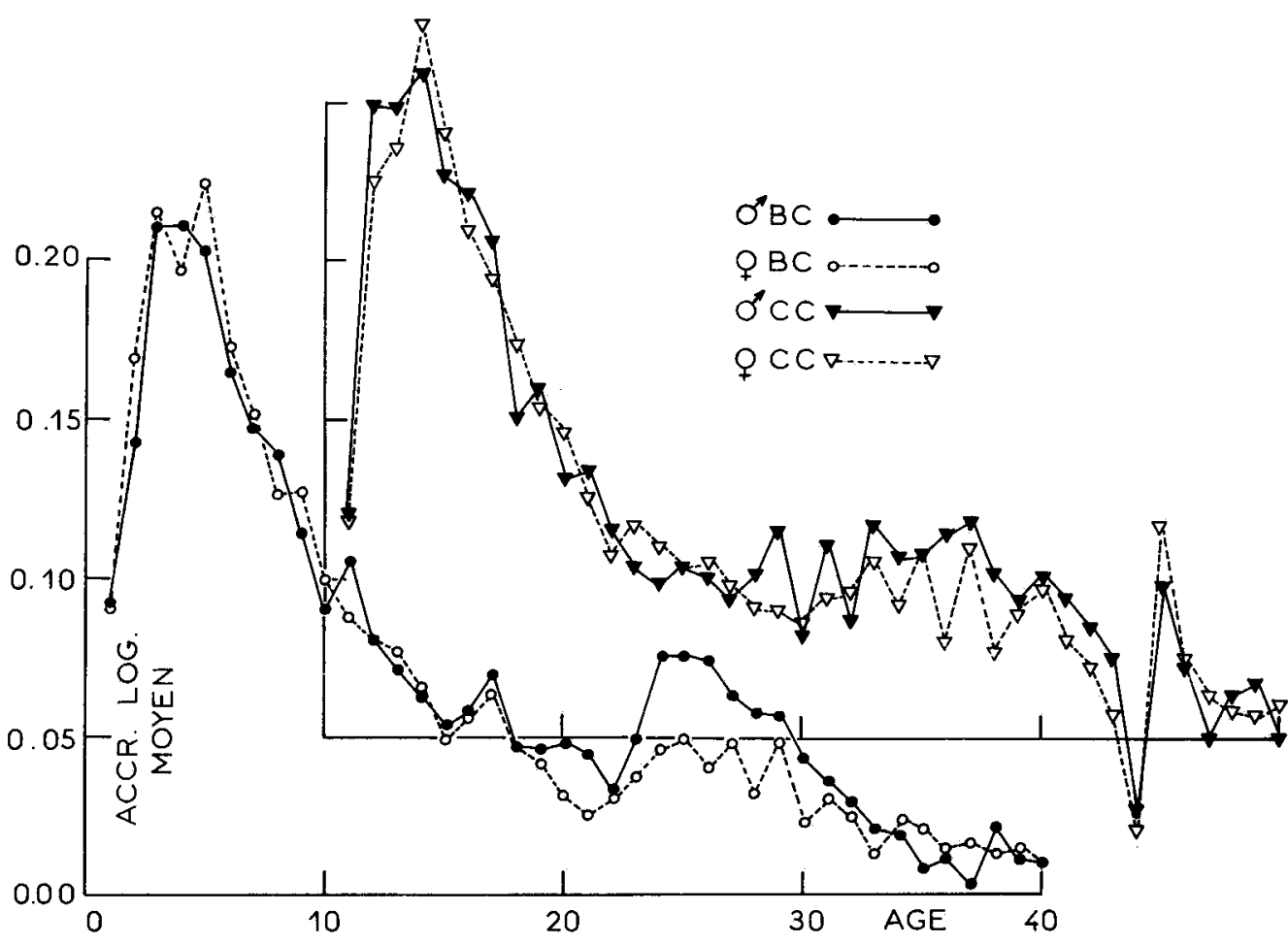

FIG. 7. - Accroissements logarithmiques moyens

A l'âge $t$ on représente la valeur moyenne $\mathrm{du} \ln \left(w_{t} / w_{t_{-1}}\right)$ où $w$ est le poids, et ce pour les groupes dont le nom est donné dans le graphique

de transition. Une fois de plus, les mâles ont une croissance plus intense que les femelles durant la seconde phase. Cette différence est plus accentuée chez les hybrides que chez les consanguins. 


\section{IV. - DISCUSSION}

\section{I.}

Dans la description des résultats nous avons distingué trois phases de croissance dont les caractéristiques permettaient de distinguer les différents groupes expérimentaux. Les courbes d'accroissements logarithmiques suggéreraient l'introduction d'une étape de transition entre l'enfance et l'adolescence. Cette étape s'étendrait entre la fin du régime lacté exclusif et le début de la maturation sexuelle. Nous donnerons ci-après une interprétation des principaux faits observés pour les deux premières phases. L'âge adulte fera l'objet d'un travail séparé.

\section{2.}

L'effet de la nourrice est considérable ainsi qu'il ressort notamment des travaux de Young et al. (r965) et de ceux de Buthrer et METrakos (1950). Selon ces derniers, l'effet nourrice serait maximum aux alentours du jour $\mathrm{I}_{4}$; ceci correspondrait à la fin de la lactation. Nous constatons également peu avant cette date un accroissement de la dispersion des courbes de croissance à l'intérieur des nichées génétíquement homogènes. Durant les deux premières semaines, les animaux supposés génétiquement identiques se comportent d'une manière très semblable chez une même nourrice, alors qu'ils montrent des courbes de croissance nettement différentes lorsqu' ils sont élevés par des nourrices différentes. Qui plus est, des souris de souche $\mathrm{C}_{3} \mathrm{H}$ et $\mathrm{C} 57 \mathrm{BL}$, ont à peu près la même croissance pendant les quatorze premiers jours, s'ils ont une même nourrice. Les hybrides, par contre, prennent le pas sur les consanguins de leur nichée. Ces résultats concordent avec les données de Youns et al. (I965).

Certains individus, malgré l'homogénéité habituelle, se distinguent de leurs homologues. La figure 4. $\mathrm{I}$ en donne un exemple: on y voit une courbe qui reste inférieure à toutes les autres. De tels animaux devraient être examinés de plus près afin de déterminer la cause de leur comportement (génétique, pathologique).

Nous trouvons encore que les hybrides ont une croissance plus rapide que les consanguins. Ce fait rejoint les observations classiques en la matière.

A l'intérieur des groupes consanguins, la variabilité est plus grande que dans les groupes d'hybrides. Toutefois à l'intérieur d'une nichée les courbes de croissance se superposent également bien dans tous les groupes. La variabilité provient donc de différences entre nichées et seraient dues aux différences entre nourrices. Il reste à savoir pourquoi l'effet nourrice est plus important chez les consanguins que chez les hybrides, et pourquoi les deux générations de consanguins sont différentes à ce point de vue.

L'aspect des courbes change notablement de la génération o à la génération $\mathrm{I}$, même lorsqu'il s'agit de souris consanguines dans les deux cas. On peut expliquer ce phénomène par un effet d'échantillonage des nourrices ou par un effet saisonnier dî̀ principalement à la nourriture, puisque les autres facteurs étaient contrôlés. Les différences sexuelles ne se manifestent pas sur les courbes de croissance superposées au moins pendant la première phase. 
4. 3 .

Les courbes de poids et d'accroissements durant la seconde phase traduisent les modifications physiologiques qui accompagnent l'adolescence. L'aspect général du phénomène est en effet celui qui a été décrit entre autres par TANNER (I955). Nous observons ici une série de faits classiques : effet du sexe, de la consanguinité et de l'hybridisme. Les différences entre les consanguins des deux générations sont plus difficiles à expliquer. On peut alléguer les mêmes raisons que pour l'enfance : l'effet du milieu serait prépondérant puisque les différences génétiques impliqueraient une conservation de la variabilité au cours des générations ce qui n'est pas vérifié. L'effet marqué du sevrage sur les $\mathrm{C}_{57} \mathrm{BL}$ et $\mathrm{BB}$ est, à notre connaisance, un fait nouveau. Il s'agit vraisemblablement d'une caractéristique génétique puisqu'elle est liée à la souche $\mathrm{C} 57 \mathrm{BL}$. Il serait intéressant de confirmer ce fait par une étude de l'hérédité de ce caractère.

\section{4 .}

Nous avons considéré dans 1'introduction que les modèles de croissance fournissaient une méthode de réduction des données à quelques paramètres. Rappelons succinctement en quoi consiste la construction habituelle des modèles de croissance. On suppose sur la base de considérations diverses que la croissance obéit à une loi qui peut s'écrire sous forme d'une équation différentielle du type (HAL,D, I952):

$$
f^{\prime}(t)=h[f(t)] \cdot g(t)
$$

où $f(t)$ est le poids considéré comme fonction du temps $t, f^{\prime}$, la dérivée de $f$, et $h$ et $g$ deux fonctions définies par ailleurs. Ia solution de l'équation (I) est, en pratique caractérisée par trois ou quatre paramètres dont l'interprétation est donnée dans le modèle. Comme d'autre part le poids n'est pas observé sans erreur, le modèle est complété en introduisant un processus $e$ tel que :

$$
w(t)=f(t)+e(t)
$$

où $w(t)$ est le poids observé à l'instant $t$ et $f$ une solution de (I). En vue d'estimer les paramètres du modèle, et donc de réduire les données à ceux-ci, on supposera que l'espérance de $e$ est partout nulle et que les variables aléatoires $e(t)$ et $e(s)$ sont toujours indépendantes pour $t$ différent de $s$. A ce prix, il est possible d'estimer les paramètres du modèle et de connaître les caractéristiques statistiques des estimateurs. L'interprétation $\mathrm{du}$ processus $e$, souvent appelé erreur, mérite un commentaire. Il ne s'agit pas seulement de représenter par $e$ les erreurs de mesure, d'ailleurs négligeables dans la plupart des expériences de croissance, mais d'exprimer que le poids lui-même oscille d'une manière aléatoire. On admet généralement que les oscillations sont dues aux fluctuations incontrôlées du milieu. Les facteurs contrôlés et les facteurs génétiques affectent les paramètres du modèle. Ceci exclut donc la possibilité de facteurs du milieu ayant une action systématique au cours de 1'expérience, tels un accroissement de la photopériode, de la température ou une variation saisonnière quelconque de la nourriture. Cette méthode a fait l'objet de commentaires critiques de la part de nombreux auteurs. Les principaux reproches adressés à ces techniques sont les suivants :

I $^{\circ}$ rien ne permet de supposer que l'équation différentielle (I) est la plus utile, ni même indiquée (HALD, I952) ; 
$2^{\circ}$ 1'ajustement même " bon ", ne suffit pas à confirmer une théorie (FELIER, I968) qui engendre la courbe ajustée;

$3^{\circ}$ les erreurs $e(t)$ ne sont pas indépendantes (RAO, I965).

Les courbes les plus usuelles ont l'aspect d'une sigmoïde et ne peuvent donc pas décrire les trois phases que nous avons relevées. Cette difficulté a déjà été signalée par LAIRD (I966) pour la phase adulte et par BACKMANN (I939). Ces auteurs et d'autres encore pallient cette lacune par l'introduction de paramètres supplémentaires dans le modèle, ce qui complique les problèmes d'estimation. L'effet nourrice est un facteur systématique et les poids sont donc corrélés entre eux. Nous trouvons ainsi une confirmation supplémentaire de la non indépendance des erreurs. Nous ne pouvons donc pas valablement estimer les paramètres des modèles sur les courbes de croissance individuelles.

\section{5 .}

Les courbes des moyennes et écarts-types sont une autre manière de résumer l'information contenue dans les données. On peut voir que la "bande noire" du graphique reproduit en général fidèlement l'allure de la courbe des moyennes. Toutefois, cette dernière doit s'interpréter avec prudence ; ainsi dans les groupes $\mathrm{C}_{3} \mathrm{H}$, la présence de deux faisceaux de courbes de poids fait que la courbe des moyennes se situe pratiquement à certains jours dans une zone où aucune courbe n'est présente. L'interprétation d'une même valeur moyenne peut donc être différente d'un groupe à l'autre, puisqu'elle ne permet pas d'inférer quant à la distribution de la variable observée. Or la distribution d'une variable peut être biologiquement plus instructive que sa moyenne. Ainsi les $\mathrm{C} 57 \mathrm{BL}$ et les $\mathrm{C} 3 \mathrm{H}$ ont sensiblement mêmes poids moyens, mais les différences entre groupes sont néanmoins importantes par les effets de groupement observés chez $\mathrm{C}_{3} \mathrm{H}$. Une remarque analogue s'applique aux écarts-types qui ne peuvent s'interpréter qu'en fonction des graphiques superposés correspondants (dispersion). Les analyses de variance très souvent pratiquées sur les poids à différents âges permettent de dégager pour ceux-ci des faits aisément observables sur les graphiques superposés par famille (importance de la nourrice, de la mère, de l'hybridisme). L'analyse de la variabilité pourrait utilement être complétée par l'étude de la matrice de covariance ou des corrélations des différentes grandeurs observéess. On peut à cet égard s'attendre à des corrélations fortes entre les poids rapprochés, puisque les oscillations des courbes de poids sont de faible amplitude, comparées aux différences entre courbes.

\section{V. - CONCLUSION}

\section{I.}

Les graphiques superposés nous ont permis de mettre en évidence un certain nombre de faits déjà connus grâce à d'autres méthodes; il s'agit de l'effet nourrice, du rôle de la consanguinité, de l'hybridisme, de l'effet sexuel dans l'adolescence, de l'absence de plateau dans le poids de l'âge adulte. Ceci montre que la technique de superposition graphique permet de visualiser les résultats d'une expérience de croissance et qu'elle fournit une première analyse qualitative des données. Nous pensons 
qu'elle pourrait être employée utilement dans les études longitudinales comme préliminaire à une étude statistique plus poussée. Nous pourrons alors exiger de l'analyse mathématique qu'elle mette au moins en évidence les faits que nous avons relevés. En outre, 1'analyse devrait fournir des mesures plus précises pour les dates de transition entre les phases, la dispersion, l'effet de groupement en faisceaux, etc.

\section{2.}

I1 serait intéressant d'effectuer des back-cross avec les individus de la génération I et d'analyser les courbes superposées par famille. Si nous supposons un effet génétique de type mendélien, nous devons nous attendre à ce que les courbes des enfants d'une même mère, élevés par une même nourrice se groupent en plusieurs faisceaux au lieu de se superposer toutes les unes aux autres.

\section{3 .}

Nous pensons en définitive que le rôle d'analyses graphiques analogues à celles que nous proposons ici est double. En tant que traitement préliminaire, elles permettent de résumer sans perte une grande masse d'information et ce de manière peu coûteuse ; elles montrent ainsi les principales caractéristiques de l'expérience et orientent vers des traitements statistiques ultérieurs. En second lieu, après les calculs, elles simplifient l'interprétation des résultats.

Reçu pour publication en juin 1972.

\section{REMERCIEMEN'TS}

Je remercie le professeur HEuTs d'avoir dirigé le présent travail et de m'avoir fourni le matériel nécessaire à sa réalisation. Les remarques et conseils du professeur LEGAY m'ont été très utiles dans la mise au point de ce texte.

\section{SUMMARY}

\section{A GRAPHICAL METHOD OF GROWTH CURVES ANALYSIS}

Mice weight curves are analyzed by means of a simple graphical method which consists in drawing several growth curves in the same graph. This technique yields a first qualitative analysis of the growth of two different inbred strains and of their hybrids. Three phases appear in the curves; they correspond to infancy, adolescence and adult age. The first two phases are described with some detail. The method is compared to oher classical methods on growth analysis.

\section{RÉFÉRENCES BIBLIOGRAPHIQUES}

Backmann G., 1939. Das Wachstum der weissen Maus. Lunds Univ. Arssk. Avd., 2, 35, I-20.

Butler L., Metrakos J. D., I950. A study of size inheritance in the house mouse. I. The effect of milk source. Can. J. Res., 28-D, i6-34.

FELLER W., I968. An introduction to probability theory and its applications, 2 ( $3^{\mathrm{e}}$ edition). Wiley, New York. 
Gall G. A. A., KylE W. H., I968. Growth of the laboratory mouse. Theoret. Appl. Genet., 88 304-308.

HALD A., 1952. Statistical theory with engineering applications, 658-662. Wiley-Toppan, New York, Tokyo.

KiDwell J. F., Howard A., I97o. The inheritance of growth and form in the mouse. III. Orthogonal polynomials. Growth, 34, 87-98.

Kidwell J. F., Howard A., Laird A. K., I 969 . The inheritance of growth and form in the mouse. II. The Gompertz equation. Growth, 33, 339-352.

LAIRD A. K., I966. Postnatal growth of birds and mammals. Growth, 30, 349-363.

LAIRD A. K., I967. Growth curves in inbred mice. Nature, 213, 796-799.

RAO C. R. 1965. The theory of least squares when the parameters are stochastic and its application to the analysis of growth. Biometrika, 52, 447-458.

TANNER J. M., I955. Growth at adolescence. Blackwell scientific publications, Oxford.

Von BertalanfFy L., 1960. Principles and theory of growth. In : Fundamental aspects of normal and malignant growth, ed. Novinsky W. W. Elsevier.

Young C. W., Legates J. E., Farthing B. R., I965. Prenatal and postnatal influences on growth, prolifacy and maternal performance in mice. Genetics, 52, 553-56I. 